\title{
Comparison of amyloid PET measured in Centiloid units with neuropathological findings in Alzheimer's disease
}

\author{
Sanka Amadoru ${ }^{1 *} \mathbb{D}$, Vincent Doré ${ }^{1,2} \mathbb{D}$, Catriona A. McLean ${ }^{3} \mathbb{D}$, Fairlie Hinton ${ }^{3}$, Claire E. Shepherd ${ }^{4} \mathbb{D}$, \\ Glenda M. Halliday ${ }^{4,5}$ (D) Cristian E. Leyton ${ }^{5}$ (D) Paul A. Yates ${ }^{1}$ (D) John R. Hodges ${ }^{5}$, Colin L. Masters ${ }^{3}$ (D), \\ Victor L. Villemagne ${ }^{1}$ (D) \\ and Christopher C. Rowe ${ }^{1}$ (D)
}

\begin{abstract}
Background: The Centiloid scale was developed to standardise the results of beta-amyloid (AB) PET. We aimed to determine the Centiloid unit (CL) thresholds for CERAD sparse and moderate-density neuritic plaques, Alzheimer's disease neuropathologic change (ADNC) score of intermediate or high probability of Alzheimer's Disease (AD), final clinicopathological diagnosis of $A D$, and expert visual read of a positive $A \beta P E T$ scan.

Methods: A $\beta$ PET results in CL for 49 subjects were compared with post-mortem findings, visual read, and final clinicopathological diagnosis. The Youden Index was used to determine the optimal $C L$ thresholds from receiver operator characteristic (ROC) curves.
\end{abstract}

Results: A threshold of $20.1 \mathrm{CL}(21.3 \mathrm{CL}$ when corrected for time to death, AUC 0.97) yielded highest accuracy in detecting moderate or frequent plaque density while $<10 \mathrm{CL}$ was optimal for excluding neuritic plaque. The threshold for ADNC intermediate or high likelihood AD was $49.4 \mathrm{CL}$ (AUC 0.98). Those cases with a final clinicopathological diagnosis of AD yielded a median CL result of 87.7 (IQR \pm 42.2 ) with $94 \%>45 \mathrm{CL}$. Positive visual read agreed highly with results $>26 \mathrm{CL}$.

Conclusions: Centiloid values $<10$ accurately reflected the absence of any neuritic plaque and $>20 \mathrm{CL}$ indicated the presence of at least moderate plaque density, but approximately $50 \mathrm{CL}$ or more best confirmed both neuropathological and clinicopathological diagnosis of Alzheimer's disease.

Keywords: Amyloid imaging, Alzheimer's disease, Centiloids, Positron emission tomography, Neuropathology

\section{Background}

Current standard-of-truth (SoT) diagnosis of Alzheimer's disease (AD) largely depends on neuropathological demonstration of brain amyloid-beta $(A \beta)$ plaques and tau tangles [1]. Recently, research criteria for detection of $\mathrm{AD}$ have emphasised the importance of amyloid and tau positron emission tomography (PET) imaging biomarkers $[2,3]$.

${ }^{11} \mathrm{C}$-Pittsburgh Compound B $\left({ }^{11} \mathrm{C}\right.$-PiB), ${ }^{18} \mathrm{~F}$-florbetaben (FBB), ${ }^{18}$ F-florbetapir, ${ }^{18} \mathrm{~F}$-flutemetamol and ${ }^{18} \mathrm{~F}$ NAV4694 (NAV) are PET tracers that demonstrate

\footnotetext{
* Correspondence: sanka.amadoru@austin.org.au

${ }^{1}$ Department of Molecular Imaging and Therapy, Austin Health, 145 Studley

Road, Heidelberg Vic. 3084, Australia

Full list of author information is available at the end of the article
}

binding to brain $\mathrm{A} \beta$ in $\mathrm{AD}$ from the preclinical $\mathrm{AD}$ stage onwards, with good sensitivity and specificity as biomarkers of antemortem $\mathrm{AD}$ pathology and predictors of progression to AD dementia [4-10]. Amyloid PET scans are used for inclusion and monitoring in AD-modifying clinical therapy trials and to aid clinical diagnosis and prognostication [11, 12].

Variability in tracers, PET scanners, procedural factors, and analysis methods across imaging centres have driven attempts for quantitative standardisation of amyloid PET results. Klunk and colleagues [13] derived a scale of "Centiloid" units (CL) for standardised reporting of amyloid imaging. CL values range beyond the "anchor-point" of 0 , representing young healthy controls, and 100, representing 
the amyloid burden present in average mild to moderate severity dementia due to $A D$. This important work allows for amyloid PET scans across different sites to yield comparable data.

Comparison of neuropathological data with positive or negative amyloid PET scans based on expert visual read has been performed for ${ }^{11} \mathrm{C}$-PiB, ${ }^{18} \mathrm{~F}$-florbetapir, ${ }^{18} \mathrm{~F}$-florbetaben and ${ }^{18} \mathrm{~F}$-flutemetamol $[7,9,14-18]$. In vivo biomarkers such as cerebrospinal fluid $A \beta$, tau PET, and volumetric MRI have been compared with amyloid PET in CL $[19,20]$. Three recent studies have examined the performance of amyloid PET CL thresholds compared with SoT neuropathology. These studies reported thresholds for detection of moderate or frequent neuritic plaque ranging from 12 to $24 \mathrm{CL}$ but did not correct for time elapsed between amyloid scan and death and had relatively few cases with CL values close to the threshold values [21-23]. Only one compared to Alzheimer's disease neuropathologic change (ADNC) rating [1] or to expert visual read report of a positive scan [21].

We aim to further define the accuracy of $\mathrm{CL}$ values when compared with SoT post-mortem neuropathological data on neuritic amyloid plaque density, ADNC rating, final clinicopathological diagnosis of $\mathrm{AD}$, and visual reading threshold for a positive amyloid PET scan.

\section{Methods}

\section{Compliance with ethical standards}

Ethics approval was obtained from the Austin Health $\mathrm{Hu}$ man Research Ethics Committee (reference LNR/17/405).

\section{Subject identification and demographic data collection}

Fifty-one subjects in total with various ante-mortem and post-mortem diagnoses were retrospectively identified by cross-referencing the databases of the Austin Health Molecular Imaging Dementia Research group, the Sydney Brain Bank, and the Victorian Brain Bank. These subjects, with prior informed consent, had undergone both an amyloid PET scan at Austin Health and post-mortem neuropathologic brain evaluation in Melbourne or Sydney between all years recorded in the database (2004 to 2017). Exclusion criteria for these prior studies were history of stroke, significant medical illness, recent cancer, and substance use disorder. Two of the 51 cases were excluded due to a diagnosis of familial AD, because different neuropathological processes in this condition, such as a significantly greater density of $A \beta$ plaques in the cerebellum than in sporadic AD $[24,25]$, may have confounded amyloid PET quantification and interpretation. Data has been published on a proportion of the cases [26, 27].

\section{Amyloid PET imaging and Centiloid determination}

$\mathrm{A} \beta$ imaging was performed with either ${ }^{11} \mathrm{C}-\mathrm{PiB}$ or ${ }^{18} \mathrm{~F}$ florbetaben (FBB). The methodology for PET imaging with these tracers has been previously described $[28,29]$. A 20-min acquisition was commenced $50 \mathrm{~min}$ postinjection of ${ }^{11} \mathrm{C}-\mathrm{PiB}$ or $90 \mathrm{~min}$ post-injection of $\mathrm{FBB}$. A transmission scan was performed for attenuation correction. PET images were reconstructed using a $3 \mathrm{D}$ rowaction maximum likelihood algorithm (RAMLA). The standard Centiloid cortical and whole cerebellar volumes of interest template were applied to the summed and spatially normalised PET images in order to obtain standardised uptake value ratios (SUVR). For this study, we used the CapAIBL software package, which when compared to standard approach has the benefit of not requiring a corresponding MRI to quantify the PET scan $[30,31]$. This package has been validated against the standard Centiloid method that uses the public domain software program SPM8 to spatially normalise each subject's MRI and then apply those parameters to spatially normalise the amyloid PET scan [30]. The SUVR were transformed into Centiloid units by linear transformation using the PET tracer-specific equations published for conversion of Centiloid method SUVR to Centiloid units with a minor correction applied for the CapAIBL registration [13, 28, 29, 32].

\section{Neuropathologic evaluation}

Neuropathological evaluation was performed at the Victorian Brain Bank (Melbourne, Australia) and Sydney Brain Bank (Neuroscience Research Australia, Sydney, Australia) to determine a global $\mathrm{C}$ score from inferior temporal regions of fixed brain hemispheres based on the Consortium for Establish a Registry for Alzheimer's Disease (CERAD) neuropathologic assessment guidelines [33]. Frequency of neuritic plaques per $\times 100$ microscopic field were categorised as none, sparse, moderate or frequent with corresponding $C$ scores of $0,1,2$ or 3 respectively, as described in published guidelines [34]. ADNC classification was also obtained as defined by the NIA-AA 2012 criteria [1]. The ADNC rating uses the Thal amyloid plaque distribution, the Braak neurofibrillary tangle stage and the CERAD neuritic plaque score, to classify $\mathrm{AD}$ neuropathologic change as not, low, intermediate or high.

\section{Visual read}

One amyloid PET expert reader (author CR), blinded to $\mathrm{CL}$ values and neuropathological data, visually interpreted all scans using MedView v12 software, viewing images in greyscale and rainbow colour scale. The method used to visually read amyloid PET has been previously described [35]. Scans were classified positive when cortical activity was equal to or greater than white matter activity in one or more lobes. 


\section{Clinicopathological diagnosis}

The clinicopathological diagnosis for each case factored in both neuropathological assessment and antemortem clinical diagnosis. Neuropathological diagnosis was made in accordance with the published guidelines [34] and included morphological examination with immunohistochemistry analyses for $\mathrm{A} \beta$, tau, TDP43 and alphasynuclein in several brain regions. There were $17 \mathrm{AD}$ and 32 non-AD cases. Non-AD cases included diagnoses of frontotemporal dementia $(n=12)$, normal controls $(n=3)$, dementia with Lewy bodies $(n=3)$, Parkinson's disease dementia with concurrent diffuse Lewy bodies $(n=3)$, hippocampal sclerosis $(n=2)$, Creutzfeldt-Jakob disease $(n=2)$, progressive supranuclear palsy $(n=2)$, motor neuron disease $(n=1)$, hippocampal ischaemia $(n=1)$, corticobasal degeneration $(n=1)$, multisystem atrophy $(n=1)$ and a case of mixed AD and dementia with Lewy bodies $(n=1)$. This last case was included in all analyses, except for the "Centiloid results in clinicopathological AD diagnosis" analysis.

\section{Statistical analyses}

Three aspects of CL performance were investigated. Firstly, $\mathrm{CL}$ values were compared with dichotomized neuropathological C score categories using two different approaches: "high vs low" plaque density ("high" = moderate and frequent, and "low" = none and sparse) and "any vs none" ("any" = sparse, moderate and frequent, and "none" = none). A Youden index [36] was used to determine the optimal CL thresholds from receiver operator characteristic curves. $\mathrm{CL}$ values were also compared with binary ADNC classification of "unlikely AD" (ADNC scores of not or low) vs "likely AD" (ADNC scores of intermediate or high). Secondly, values were compared with visual read (positive or negative). Thirdly, CL values were compared with cases of $\mathrm{AD}$ as determined by clinicopathological diagnosis using descriptive statistics. To assess for the contribution of interval from PET scan to time to death, analyses were repeated using adjusted $\mathrm{CL}$ values, after applying a sigmoidal adjustment derived from our previous work [37]. We derived that $\mathrm{CL}$ increases very slowly below $20 \mathrm{CL}$, then accelerates to a maximum of $5 \mathrm{CL}$ increase per year for the almost linear mid-section ( 40 to $110 \mathrm{CL}$ ) of the sigmoid curve that best describes amyloid accumulation over time, before slowing again at higher $\mathrm{CL}$ values. Consequently, each individual $\mathrm{CL}$ value was adjusted to that expected at the time of death based on the average rate of increase for the CL level at the time of the scan and the duration between the scan and post-mortem examination.

\section{Results}

\section{Case characteristics}

Of the 49 included subjects, 33 underwent ${ }^{11} \mathrm{C}$ PiB PET and 16 underwent FBB PET. Thirty-eight (78\%) cases were male. The mean age at death was 76 years, and the median interval between date of last amyloid PET scan and death was 2.75 years (IQR \pm 3.05 , range 0.03 to 5.64 ). Twenty-five subjects had a $\mathrm{C}$ score of 0 (no plaques), five had a $\mathrm{C}$ score of 1 (sparse plaques), five had a $\mathrm{C}$ score of 2 (moderate plaques) and 14 had a score of 3 (frequent plaques). Twenty had ADNC classification of intermediate or high neuropathologic change.

\section{Centiloid results and neuropathological C score categories}

There were 19 (39\%) patients with "high" and 30 (61\%) with "low" C scores. The receiver operator characteristic curve demonstrated an optimal threshold of 20.1 CL for detection of a high level of amyloid plaque (i.e. moderate or frequent neuritic plaques). After applying the sigmoidal adjustment for interval from scan to post-mortem, the putative optimal threshold was 21.3 CL (Fig. 1a). Data points and threshold are shown in Fig. 2a.

When neuritic plaque scores were grouped as "any" (i.e. sparse or more) vs "none", there were 24 (49\%) cases with "any" and 25 (51\%) with no neuritic plaques. The optimal CL threshold found for detecting the presence of any amyloid plaques (i.e. sparse or more) was 9.5 CL. After applying the sigmoidal adjustment for interval from scan to post-mortem, the putative optimal threshold was 9.6 CL (Fig. 1b). Data points and threshold are shown in Fig. $2 b$.

\section{Centiloid results and ADNC diagnosis}

There were $20(41 \%)$ patients with intermediate to high probability of AD. The optimal threshold found for detection of intermediate to high ADNC was $46.9 \mathrm{CL}$. After applying the sigmoidal adjustment for interval from scan to post-mortem, the putative optimal threshold was 49.4 CL (Fig. 1c). Data points and threshold are shown in Fig. 2c.

\section{Centiloid results and amyloid PET visual read}

Correlation of Centiloid values with amyloid PET expert visual read (positive or negative) yielded an AUC of 1.0 and optimal CL threshold of 26. Using this threshold, there was $100 \%$ agreement between Centiloid (elevated/ not elevated) and visual read (positive/negative) (Fig. 3). The effect of the visual read threshold which equates to $26 \mathrm{CL}$ for detection of moderate or frequent plaque compared to the quantification best threshold of 20.1 $\mathrm{CL}$ is a minor reduction from 1.0 to 0.95 in sensitivity and from 1.0 to 0.90 in specificity.

\section{Centiloid results in clinicopathological AD diagnosis}

$\mathrm{CL}$ values were reviewed for the 17 cases that had a final clinicopathological diagnosis of AD. The median CL result was 87.7 ( $\mathrm{IQR} \pm 42.2$ ), and 16 of the 17 cases (> 


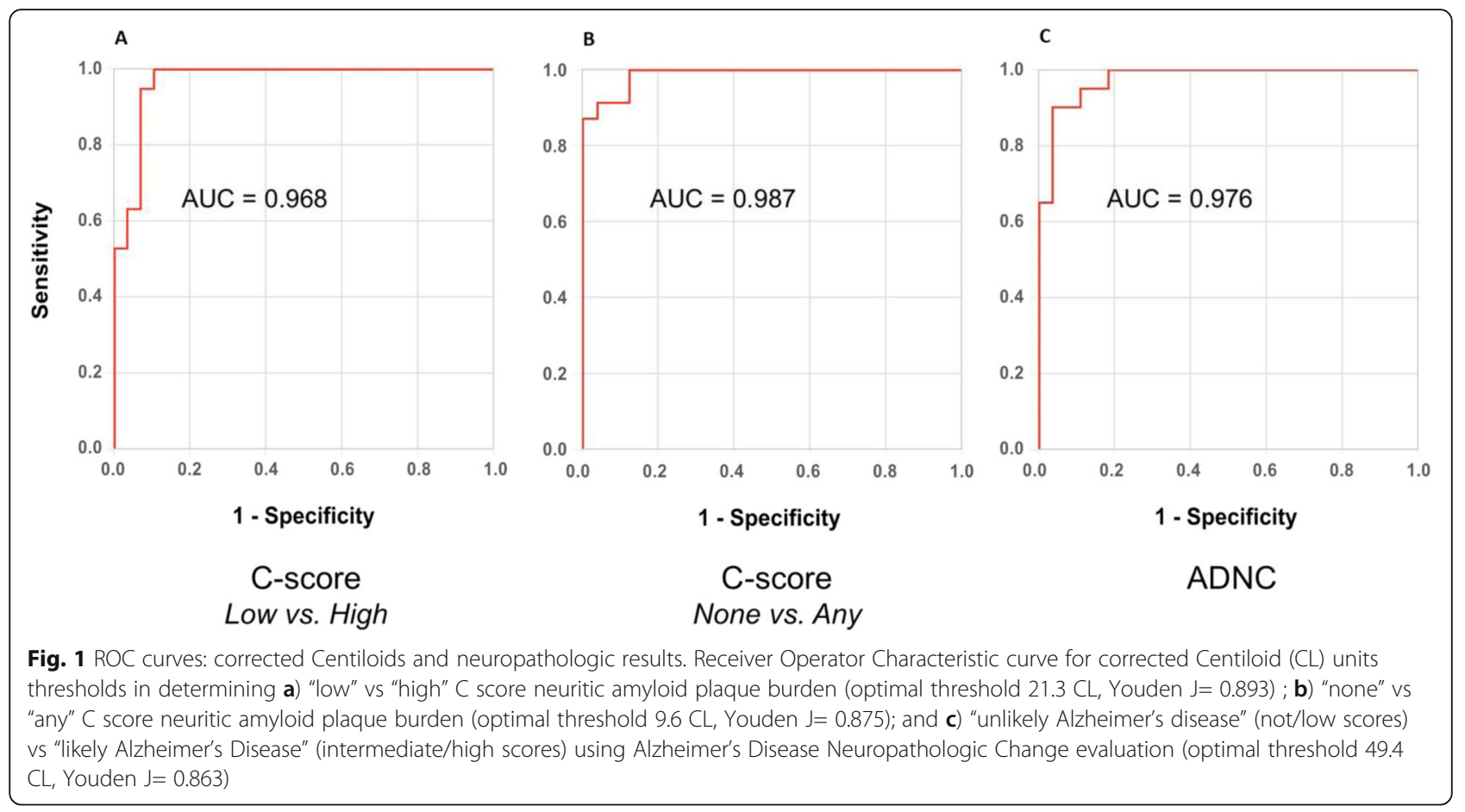

90\%) had a value of $>45 \mathrm{CL}$. In contrast, the median CL result in the 11 cases with a clinicopathological diagnosis of FTD was -4.9 CL.

\section{Discussion}

Improving accuracy of the detection of brain amyloid plaques is important for clinical trial enrichment in the quest to develop disease-modifying or curative treatment for the growing burden of Alzheimer's disease. Contemporary clinical application can also better assist diagnosis, prognostication and planning for patients with cognitive disorders.

We have demonstrated that a threshold of $20.1 \mathrm{CL}$ was optimal for the detection of "high" levels of neuritic plaque density, as determined by $\mathrm{C}$ score moderate or frequent classification. In other words, values

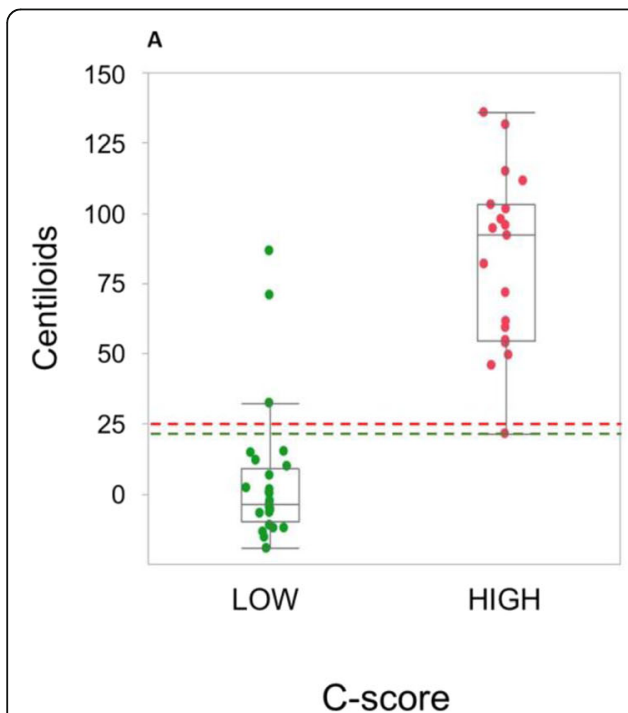

B

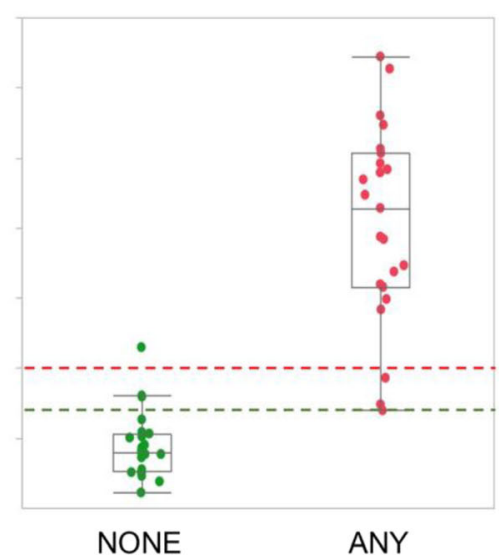

C-score
C

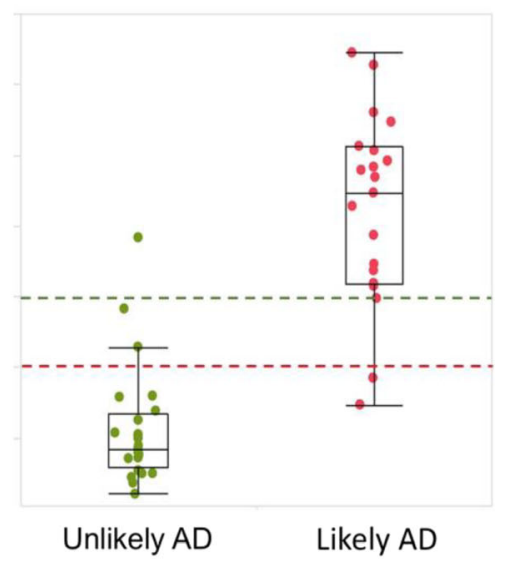

ADNC

Fig. 2 Scatterplots: corrected Centiloids and neuropathologic results. Scatterplots for corrected Centiloids against a) "low" vs "high" C score neuritic amyloid plaque burden, b) "none vs "any" C score neuritic plaque burden, and c) "unlikely AD" and "likely AD" using Alzheimer's Disease Neuropathologic Change evaluation scores. The red dashed line denotes the 25 Centiloid (CL) mark, and the green dashed lines denote the thresholds of A) $21.3 \mathrm{CL}$; B) $9.6 \mathrm{CL}$; and C) $49.4 \mathrm{CL}$ 


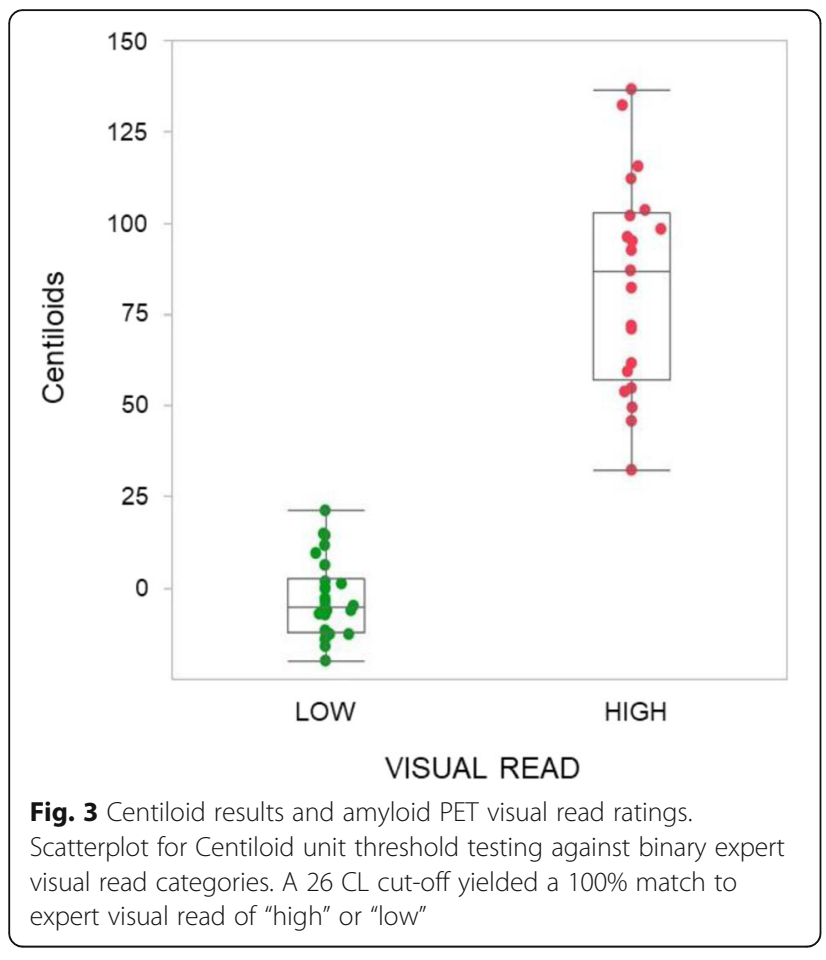

of 20.1 CL or lower accurately reflected the absence of moderate or frequent plaques, with a high AUC. This threshold was not significantly altered when corrected for time between scan and post-mortem. CL results below this threshold should provide reassurance that patients are unlikely to have Alzheimer's disease. This is reasonably concordant with the findings of Navitsky and colleagues, who determined a threshold of 24.1 CL with florbetapir for CERAD amyloid plaque classification of moderate or frequent vs sparse or none, in 59 individuals [22]. This is also concordant with the Centiloid analysis by Dore and colleagues of a florbetaben phase III post-mortem study in 66 individuals, which yielded a threshold of 19 CL for the same categorisation [23]. These thresholds are higher than those identified by La Joie et al., who determined a threshold of 12.2 CL for separating none or sparse plaques from moderate or frequent plaques; but oddly they found the same threshold for separating any plaques from no plaques in 179 individuals scanned with ${ }^{11} \mathrm{C}-\mathrm{PiB}$ PET [21]. Those authors did, however, suggest $24 \mathrm{CL}$ to be a more appropriate threshold for "identifying clinically meaningful $A \beta$ burden" based on ADNC neuropathological criteria and Thal scores. These thresholds may be useful as cut-offs for clinical trial enrichment, or for guiding decisionmaking about commencing potential disease-modifying therapies when they become available.

For the detection of "any" amyloid plaque (sparse or more), the optimal threshold identified was 9.5 CL. Once again, this value only marginally increased when corrected for time between scan and post-mortem. This threshold is similar to two standard deviations of young controls as determined by Klunk and colleagues, equalling 8.68 CL [13] and 12.2 CL [21] for PiB and FBB respectively. This suggests there are no differences in amyloid tracer binding between young and old individuals when no amyloid plaques are present, indicating no substantial increase in non-specific binding of these tracers nor significant changes in tracer kinetics with normal ageing.

A threshold of $26 \mathrm{CL}$ exactly matched expert visual read of positive vs negative scan. This is consistent with good concordance (97\%) noted by Leuzy and colleagues [19] between visual read and PiB CL results. This visual threshold is slightly higher than the CL threshold for neuropathology indicating that some persons with significant $\mathrm{AD}$ pathology will have a visually negative scan and that scan quantification is of value when identifying persons with $\mathrm{AD}$ pathology for clinical trials.

Our expert visual reader results are comparable to those reported in several phase III trials of PET tracers that compared blinded expert visual reads of amyloid imaging with post-mortem data, where moderate to frequent neuritic plaques (a "high" classification in our study) were considered positive. Florbetaben visual reads were reported as having sensitivity of $97.9 \%$ and specificity of $88.9 \%$ [16]. Florbetapir visual reads yielded sensitivity of $92 \%$ and specificity of $100 \%$ within a 2 -year window between imaging and autopsy [15]. Flutemetamol visual reads in one study correlated with both original and modified CERAD criteria, yielded respective sensitivities of $91.9 \%$ and $90.8 \%$, and specificities of $87.0 \%$ and $90.0 \%$ [17]. Three expert readers in La Joie's group [21] had 99\% accuracy for scans above a threshold of $24 \mathrm{CL}$. When considering visual read, there are rare cases of clear focal amyloid uptake that may lead to a positive visual read but a low CL score but there were no such cases in our cohort.

In our cases with clinicopathological $\mathrm{AD}$, a median $\mathrm{CL}$ value of 87.7 was found at post-mortem, but with significant variability as demonstrated by the IQR of $\pm 42.2 \mathrm{CL}$. Only one of these cases, scoring 20.1 CL (21.3 CL when corrected for time to death), returned a result under 45 $\mathrm{CL}$, suggesting that a sensitivity cut-off for defining clinicopathological AD should be considerably higher than that for detecting "high" amyloid plaques alone. This agrees well with our finding of $49 \mathrm{CL}$ as the optimal threshold for identifying cases that meet current neuropathological criteria for $\mathrm{AD}$ based on a comprehensive post-mortem brain examination, i.e. intermediate or high Alzheimer's Disease Neuropathic Change (ADNC). Our exceptional case with only $21 \mathrm{CL}$ had logopenic aphasia and 5.6 years between ${ }^{11} \mathrm{C}-\mathrm{PiB}$ scan and death at which time frequent plaques were found. The $\mathrm{PiB}$ scan was reviewed and showed mild patchy cortical binding. The 
long interval between scan and post-mortem is noteworthy and raises the possibility of more rapid than usual plaque accumulation so that the correction for time elapsed from scan did not provide an accurate estimation of the plaque burden at the time of death. Our CL findings in clinicopathological $\mathrm{AD}$ compare to estimates obtained without pathological confirmation of AD. For instance, Leuzy et al. [19] demonstrated median PiB PET results of 47.5 CL for mild cognitive impairment and 84.1 CL for $\mathrm{AD}$ in their cohorts. We reviewed the 230 patients with a diagnosis of probable AD made by a clinical panel blinded to biomarker findings including amyloid PET in the Australian Imaging Biomarkers and Lifestyle study of ageing. The mean CL in these patients was $95 \pm 30$. Larger numbers in future studies would help confirm if a clinicopathological diagnosis of AD is indeed rare when under $45 \mathrm{CL}$.

The sex distribution in this study was predominantly male at $78 \%$. No explanation for this is evident in our study, but a predominance of males was also reported in two of the three published CL neuropathological correlation studies [21, 22].

Our study adds further confirmation of these thresholds in a field where there are relatively low numbers of post-mortem results for correlation, especially for intermediate CL values. Additionally, we have adjusted for amyloid accumulation during the time between scan and post-mortem. Finally, only La Joie and colleagues [21] have also included a visual read comparison in a cohort with neuropathological assessment.

\section{Limitations}

A limitation of the study is the data distribution, in that only two subjects had results between 15 and $35 \mathrm{CL}$, consequently restricting the ability to tightly define thresholds. Specifically, these subjects had results of 20.1 and 30.9 CL.

Another limitation is the time elapsed between scan and death. This averaged approximately 3 years. We have accounted for this using a correction based on the published curve for amyloid accumulation. However, this correction had minimal effect on the study findings. Familial AD cases were excluded from this study due to potential presence of neuritic amyloid plaques in the cerebellum [24], and the lower affinity of PiB to "cotton wool" plaques found in some presenilin mutations [38]. These cerebellar plaques could interfere with the scaling to SUVR and return misleading low CL results not applicable to typical sporadic AD [25]. Separate characterisation of CL performance in familial AD is warranted.

\section{Conclusions}

In our cohort, values $<9.5 \mathrm{CL}$ accurately reflected the absence of any neuritic plaques, and $>20.1 \mathrm{CL}$ indicated the presence of at least moderate plaque density. These neuropathology-based Centiloid thresholds may be used to exclude a diagnosis of $\mathrm{AD}$ and to define groups for early intervention and other disease specific trials. Approximately $50 \mathrm{CL}$ or more best confirmed both neuropathological and clinicopathological diagnosis of Alzheimer's disease.

\section{Supplementary information}

Supplementary information accompanies this paper at https://doi.org/10. 1186/s13195-020-00587-5.

Additional file 1. Supplementary data tables.

\begin{abstract}
Abbreviations
${ }^{11} \mathrm{C}$-PiB: ${ }^{11} \mathrm{C}$-Pittsburgh Compound B; AD: Alzheimer's disease;

ADNC: Alzheimer's disease neuropathologic change; AUC: Area under the curve; $A \beta$ : Beta-amyloid; CERAD: Consortium for Establish a Registry for Alzheimer's Disease; CL: Centiloid; FBB: ${ }^{18}$ F-florbetaben; IQR: Interquartile range; MRI: Magnetic resonance imaging; NAV: ${ }^{18} \mathrm{~F}-\mathrm{NAV} 4694$ (NAV);

PET: Positron emission tomography; RAMLA: Row-action maximum likelihood algorithm; ROC: Receiver operator characteristic; SOT: Standard of truth; SUVR: Standardised uptake value ratio; TDP43: TAR DNA-binding protein 43
\end{abstract}

\section{Acknowledgements}

Not applicable.

\section{Funding}

Austin Health and Florey Institute of Neuroscience and Mental Health researchers are supported by governmental funding.

The Sydney Brain Bank is supported by the University of New South Wales and Neuroscience Research Australia.

The Victorian Brain Bank is supported by The Florey Institute of Neuroscience and Mental Health, The Alfred and the Victorian Forensic Institute of Medicine and funded in part by Parkinson's Victoria, MND Victoria, FightMND and Yulgilbar Foundation.

This imaging and prospective tissue donation for research from the Sydney cohort was supported by funding to ForeFront, a collaborative research group dedicated to the study of frontotemporal dementia and motor neurodegenerative diseases, from the National Health and Medical Research Council of Australia (NHMRC) program (\#1037746) and project (\#630489) grants and the Australian Research Council Centre of Excellence in Cognition and its Disorders Memory Node (\#CE110001021).

\section{Availability of data and materials}

Data analysed in this study have been included as supplementary information and are available from the corresponding author on request.

\section{Authors' contributions}

Sanka Amadoru contributed to the study design, data acquisition and analysis, manuscript drafting and revision for intellectual content. Vincent Dore had a major role in the data acquisition and analysis and revised the manuscript for intellectual content. Catriona McLean had a major role in the data acquisition and interpretation and revised the manuscript for intellectual content. Fairlie Hinton had a role in the data acquisition and revised the manuscript for intellectual content. Claire Shepherd had a major role in the data acquisition and interpretation and revised the manuscript for intellectual content. Glenda Halliday had a major role in the data acquisition and interpretation and revised the manuscript for intellectual content. Cristian E. Leyton had a role in the data acquisition, data analysis and interpretation and revised the manuscript for intellectual content. Paul Yates had a role in the data acquisition, data analysis and interpretation and revised the manuscript for intellectual content. John Hodges had a minor role in the data acquisition and revised the manuscript for intellectual content. Colin L Masters had a minor role in the data acquisition and revised the manuscript for intellectual content. Victor $L$ Villemagne had a major role in the data acquisition, analysis and interpretation and revised the manuscript for intellectual content. Christopher C Rowe had a major role in the study design, data acquisition, analysis and interpretation, and revised 
the manuscript for intellectual content. The author(s) read and approved the final manuscript.

\section{Author's information}

Not applicable.

\section{Ethics approval and consent to participate}

All procedures performed in studies involving human participants were in accordance with the Austin Health Human Research Ethics Committee (reference LNR/17/405) and with the 1964 Helsinki Declaration and its later amendments or comparable ethical standards.

\section{Consent for publication}

All subjects had given consent for their data to be used for research purposes.

\section{Competing interests}

- Dr. Amadoru is site principal investigator for the AbbVie ABBV-8E12 AWARE study at Austin Health. He receives a government hospital award wage for his Austin Health clinical and research appointments.

- Dr. Doré reports no relevant disclosures.

- Prof. McLean reports no relevant disclosures.

- Ms. Hinton reports no relevant disclosures.

- Dr. Shepherd is the Director of the Sydney Brain Bank, which is funded by Neuroscience Research Australia and the University of New South Wales.

- Prof. Halliday is supported by a NHMRC Senior Principal Research Fellowship (\#1079679).

- Dr. Leyton is funded by an NHMRC dementia fellowship (APP1102969).

- Dr. Yates is principal investigator on pharma-funded clinical trials including Novartis and Amgen and has received research funding from the Dementia Collaborative Research Centres (DCRC) and Eastern Melbourne Primary Healthcare Network (EMPHN).

- Prof. Hodges is supported by a NHMRC Senior Principal Research Fellowship (\#1079679) and receives royalties from Oxford University Press related to book publication.

- Prof. Masters reports no relevant disclosures.

- Prof. Rowe reports no relevant disclosures.

- Prof Villemagne reports consultancies for Lundbeck, Hoffmann La Roche and Shanghai Green Valley Co., Ltd. and speaking honoraria from Eli Lilly and Company, GE Healthcare and Fundació ACE (Barcelona), all outside the submitted work.

\section{Author details}

'Department of Molecular Imaging and Therapy, Austin Health, 145 Studley Road, Heidelberg Vic. 3084, Australia. ${ }^{2}$ CSIRO Health and Biosecurity, Parkville, Victoria 3052, Australia. ${ }^{3}$ Victorian Brain Bank, The Florey Institute of Neuroscience and Mental Health, Melbourne, Australia. ${ }^{4}$ Sydney Brain Bank, Neuroscience Research Australia and Faculty of Medicine, University of NSW, Sydney, Australia. ${ }^{5}$ The Brain and Mind Centre, Faculty of Medicine and Health, University of Sydney, Sydney, Australia.

\section{Received: 6 November 2019 Accepted: 13 February 2020} Published online: 04 March 2020

\section{References}

1. Hyman BT, Phelps CH, Beach TG, Bigio EH, Cairns NJ, Carrillo MC, et al. National Institute on Aging-Alzheimer's Association guidelines for the neuropathologic assessment of Alzheimer's disease. Alzheimers Dement 2012;8:1-13. https://doi.org/10.1016/j.jalz.2011.10.007.

2. Dubois B, Feldman $\mathrm{HH}$, Jacova $\mathrm{C}$, Hampel H, Molinuevo JL, Blennow K, et al. Advancing research diagnostic criteria for Alzheimer's disease: the IWG-2 criteria. Lancet Neurol. 2014;13:614-29. https://doi.org/10.1016/S14744422(14)70090-0.

3. Jack CR Jr, Bennett DA, Blennow K, Carrillo MC, Dunn B, Haeberlein SB, et al. NIA-AA research framework: toward a biological definition of Alzheimer's disease. Alzheimers Dement. 2018;14:535-62. https://doi.org/10.1016/j.jalz. 2018.02.018.
4. Wiley CA, Lopresti BJ, Venneti S, Price J, Klunk WE, DeKosky ST, et al. Carbon 11-labeled Pittsburgh compound B and carbon 11-labeled (R)-PK11195 positron emission tomographic imaging in Alzheimer disease. Arch Neurol. 2009;66:60-7. https://doi.org/10.1001/archneurol.2008.511.

5. Sperling RA, Johnson KA, Doraiswamy PM, Reiman EM, Fleisher AS, Sabbagh MN, et al. Amyloid deposition detected with florbetapir F 18 ((18)F-AV-45) is related to lower episodic memory performance in clinically normal older individuals. Neurobiol Aging. 2013;34:822-31. https://doi.org/10.1016/j. neurobiolaging.2012.06.014.

6. Doraiswamy PM, Sperling RA, Johnson K, Reiman EM, Wong TZ, Sabbagh MN, et al. Florbetapir F 18 amyloid PET and 36-month cognitive decline: a prospective multicenter study. Mol Psychiatry. 2014;19:1044-51. https://doi. org/10.1038/mp.2014.9.

7. Beckett TL, Webb RL, Niedowicz DM, Holler CJ, Matveev S, Baig I, et al. Postmortem Pittsburgh compound B (PiB) binding increases with Alzheimer's disease progression. J Alzheimers Dis. 2012;32:127-38. https:// doi.org/10.3233/JAD-2012-120655.

8. Sabri O, Seibyl J, Rowe C, Barthel H. Beta-amyloid imaging with florbetaben. Clin Transl Imaging. 2015;3:13-26. https://doi.org/10.1007/s40336-015-0102-6.

9. Thurfjell L, Lilja J, Lundqvist R, Buckley C, Smith A, Vandenberghe R, et al. Automated quantification of 18F-flutemetamol PET activity for categorizing scans as negative or positive for brain amyloid: concordance with visual image reads. J Nucl Med. 2014;55:1623-8. https://doi.org/10.2967/jnumed.114.142109.

10. Rowe CC, Pejoska S, Mulligan RS, Jones G, Chan JG, Svensson S, et al. Headto-head comparison of 11C-PiB and 18F-AZD4694 (NAV4694) for betaamyloid imaging in aging and dementia. J Nucl Med. 2013;54:880-6. https://doi.org/10.2967/jnumed.112.114785.

11. Schonecker S, Prix C, Raiser T, Ackl N, Wlasich E, Stenglein-Krapf G, et al. Amyloid positron-emission-tomography with [18 F]-florbetaben in the diagnostic workup of dementia patients. Nervenarzt. 2017:88:156-61. https://doi.org/10.1007/s00115-016-0249-z.

12. Johnson KA, Minoshima S, Bohnen NI, Donohoe KJ, Foster NL, Herscovitch $P$, et al. Appropriate use criteria for amyloid PET: a report of the Amyloid Imaging Task Force, the Society of Nuclear Medicine and Molecular Imaging, and the Alzheimer's Association. Alzheimers Dement. 2013;9:e-116. https://doi.org/10.1016/j.jalz.2013.01.002.

13. Klunk WE, Koeppe RA, Price JC, Benzinger TL, Devous MD Sr, Jagust WJ, et al. The Centiloid Project: standardizing quantitative amyloid plaque estimation by PET. Alzheimers Dement. 2015;11:1-15 e1-4. https://doi.org/ 10.1016/j.jalz.2014.07.003.

14. Choi SR, Schneider JA, Bennett DA, Beach TG, Bedell BJ, Zehntner SP, et al. Correlation of amyloid PET ligand florbetapir F 18 binding with Abeta aggregation and neuritic plaque deposition in postmortem brain tissue. Alzheimer Dis Assoc Disord. 2012;26:8-16. https://doi.org/10.1097/WAD. Ob013e31821300bc

15. Clark CM, Pontecorvo MJ, Beach TG, Bedell BJ, Coleman RE, Doraiswamy PM, et al. Cerebral PET with florbetapir compared with neuropathology at autopsy for detection of neuritic amyloid-beta plaques: a prospective cohort study. Lancet Neurol. 2012;11:669-78. https://doi.org/10.1016/S14744422(12)70142-4.

16. Sabri O, Sabbagh MN, Seibyl J, Barthel H, Akatsu H, Ouchi Y, et al. Florbetaben PET imaging to detect amyloid beta plaques in Alzheimer's disease: phase 3 study. Alzheimers Dement. 2015;11:964-74. https://doi.org/ 10.1016/j.jalz.2015.02.004.

17. Salloway S, Gamez JE, Singh U, Sadowsky CH, Villena T, Sabbagh MN, et al. Performance of [(18)F] flutemetamol amyloid imaging against the neuritic plaque component of CERAD and the current (2012) NIA-AA recommendations for the neuropathologic diagnosis of Alzheimer's disease. Alzheimers Dement (Amst). 2017;9:25-34. https://doi.org/10.1016/j.dadm. 2017.06.001.

18. Curtis C, Gamez JE, Singh U, Sadowsky CH, Villena T, Sabbagh MN, et al. Phase 3 trial of flutemetamol labeled with radioactive fluorine 18 imaging and neuritic plaque density. JAMA Neurol. 2015;72:287-94. https://doi.org/ 10.1001/jamaneurol.2014.4144.

19. Leuzy A, Chiotis K, Hasselbalch SG, Rinne JO, de Mendonca A, Otto M, et al. Pittsburgh compound B imaging and cerebrospinal fluid amyloid-beta in a multicentre European memory clinic study. Brain. 2016;139:2540-53. https:// doi.org/10.1093/brain/aww160.

20. Jack CR Jr, Wiste HJ, Weigand SD, Therneau TM, Knopman DS, Lowe V, et al. Age-specific and sex-specific prevalence of cerebral beta-amyloidosis, tauopathy, and neurodegeneration in cognitively unimpaired individuals 
aged 50-95 years: a cross-sectional study. Lancet Neurol. 2017;16:435-44 https://doi.org/10.1016/S1474-4422(17)30077-7.

21. La Joie R, Ayakta N, Seeley WW, Borys E, Boxer AL, DeCarli C, et al. Multisite study of the relationships between antemortem [(11)C]PIB-PET Centiloid values and postmortem measures of Alzheimer's disease neuropathology. Alzheimers Dement. 2018; https://doi.org/10.1016/j.jalz.2018.09.001.

22. Navitsky M, Joshi AD, Kennedy I, Klunk WE, Rowe CC, Wong DF, et al. Standardization of amyloid quantitation with florbetapir standardized uptake value ratios to the Centiloid scale. Alzheimers Dement. 2018; https:// doi.org/10.1016/j.jalz.2018.06.1353.

23. Dore V, Bullich S, Rowe CC, Bourgeat P, Konate S, Sabri O, et al. Comparison of (18)F-florbetaben quantification results using the standard Centiloid, MRbased, and MR-less CapAIBL((R)) approaches: validation against histopathology. Alzheimers Dement. 2019;15:807-16. https://doi.org/10. 1016/j.jalz.2019.02.005.

24. Mann DM, Pickering-Brown SM, Takeuchi A, Iwatsubo T. Members of the Familial Alzheimer's Disease Pathology Study G. Amyloid angiopathy and variability in amyloid beta deposition is determined by mutation position in presenilin-1-linked Alzheimer's disease. Am J Pathol. 2001;158:2165-75.

25. Klunk WE, Price JC, Mathis CA, Tsopelas ND, Lopresti BJ, Ziolko SK, et al. Amyloid deposition begins in the striatum of presenilin-1 mutation carriers from two unrelated pedigrees. J Neurosci. 2007;27:6174-84. https://doi.org/ 10.1523/JNEUROSCI.0730-07.2007.

26. Tan RH, Kril JJ, Yang Y, Tom N, Hodges JR, Villemagne VL, et al. Assessment of amyloid beta in pathologically confirmed frontotemporal dementia syndromes. Alzheimers Dement (Amst). 2017;9:10-20. https://doi.org/10. 1016/.j.dadm.2017.05.005.

27. Leyton CE, Villemagne VL, Savage S, Pike KE, Ballard KJ, Piguet O, et al. Subtypes of progressive aphasia: application of the international consensus criteria and validation using beta-amyloid imaging. Brain. 2011;134:3030-43. https://doi.org/10.1093/brain/awr216.

28. Rowe CC, Dore V, Jones G, Baxendale D, Mulligan RS, Bullich S, et al. 18FFlorbetaben PET beta-amyloid binding expressed in Centiloids. Eur J Nucl Med Mol Imaging. 2017;44:2053-9. https://doi.org/10.1007/s00259-017-3749-6.

29. Rowe CC, Jones G, Dore V, Pejoska S, Margison L, Mulligan RS, et al. Standardized expression of 18F-NAV4694 and 11C-PiB beta-amyloid PET results with the Centiloid scale. J Nucl Med. 2016;57:1233-7. https://doi.org/ 10.2967/jnumed.115.171595.

30. Bourgeat P, Villemagne VL, Dore V, Brown B, Macaulay SL, Martins R, et al. Comparison of MR-less PiB SUVR quantification methods. Neurobiol Aging 2015;36(Suppl 1):S159-66. https://doi.org/10.1016/..neurobiolaging.2014.04. 033.

31. Zhou L, Salvado O, Dore V, Bourgeat P, Raniga P, Macaulay SL, et al. MR-less surface-based amyloid assessment based on 11C PiB PET. PLoS One. 2014;9: e84777. https://doi.org/10.1371/journal.pone.0084777.

32. Bourgeat P, Dore V, Fripp J, Ames D, Masters CL, Salvado O, et al. Implementing the centiloid transformation for (11)C-PiB and beta-amyloid (18)F-PET tracers using CapAIBL. Neuroimage. 2018;183:387-93. https://doi. org/10.1016/.neuroimage.2018.08.044

33. Mirra SS, Heyman A, McKeel D, Sumi SM, Crain BJ, Brownlee LM, et al. The consortium to establish a registry for Alzheimer's disease (CERAD). Part II. Standardization of the neuropathologic assessment of Alzheimer's disease. Neurology. 1991;41:479-86.

34. Montine TJ, Phelps CH, Beach TG, Bigio EH, Cairns NJ, Dickson DW, et al. National Institute on Aging-Alzheimer's Association guidelines for the neuropathologic assessment of Alzheimer's disease: a practical approach Acta Neuropathol. 2012;123:1-11. https://doi.org/10.1007/s00401-011-0910-3.

35. Rowe CC, Villemagne VL. Brain amyloid imaging. J Nucl Med Technol. 2013; 41:11-8. https://doi.org/10.2967/jnumed.110.076315.

36. Ruopp MD, Perkins NJ, Whitcomb BW, Schisterman EF. Youden index and optimal cut-point estimated from observations affected by a lower limit of detection. Biom J. 2008;50:419-30. https://doi.org/10.1002/bimj.200710415.

37. Villemagne VL, Burnham S, Bourgeat $P$, Brown B, Ellis KA, Salvado O, et al. Amyloid beta deposition, neurodegeneration, and cognitive decline in sporadic Alzheimer's disease: a prospective cohort study. Lancet Neurol. 2013:12:357-67. https://doi.org/10.1016/S1474-4422(13)70044-9.

38. Ikonomovic MAE, Price J, Mathis M, Klunk W. Evaluation of cotton wool plaques using amyloid binding compounds and $A \beta$ immunohistochemistry: implications for PiB PET imaging. (abst). Human Amyloid Imaging Handbook; 2014. p. 41

\section{Publisher's Note}

Springer Nature remains neutral with regard to jurisdictional claims in published maps and institutional affiliations.
Ready to submit your research? Choose BMC and benefit from:

- fast, convenient online submission

- thorough peer review by experienced researchers in your field

- rapid publication on acceptance

- support for research data, including large and complex data types

- gold Open Access which fosters wider collaboration and increased citations

- maximum visibility for your research: over $100 \mathrm{M}$ website views per year

At $\mathrm{BMC}$, research is always in progress.

Learn more biomedcentral.com/submissions 


\section{University Library}

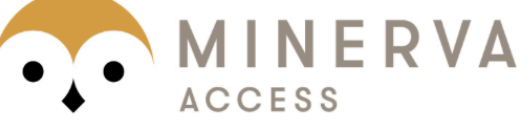

A gateway to Melbourne's research publications

Minerva Access is the Institutional Repository of The University of Melbourne

Author/s:

Amadoru, S;Dore, V;McLean, CA;Hinton, F;Shepherd, CE;Halliday, GM;Leyton, CE;Yates, PA;Hodges, JR;Masters, CL;Villemagne, VL;Rowe, CC

Title:

Comparison of amyloid PET measured in Centiloid units with neuropathological findings in Alzheimer's disease

Date:

2020-03-04

Citation:

Amadoru, S., Dore, V., McLean, C. A., Hinton, F., Shepherd, C. E., Halliday, G. M., Leyton, C. E., Yates, P. A., Hodges, J. R., Masters, C. L., Villemagne, V. L. \& Rowe, C. C. (2020). Comparison of amyloid PET measured in Centiloid units with neuropathological findings in Alzheimer's disease. ALZHEIMERS RESEARCH \& THERAPY, 12 (1), https://doi.org/10.1186/ s13195-020-00587-5.

Persistent Link:

http://hdl.handle.net/11343/245885

License:

CC BY 